\title{
Respiratory toxicity of Aspergillus versicolor: the most common indoor mould in Slovakia
}

\author{
E. Piecková ${ }^{1}$, M. Hurbánková ${ }^{1}$, S. Černá ${ }^{1}$, M. Majorošová ${ }^{1}$, \\ Z. Kováčiková ${ }^{1} \&$ D. Pangallo ${ }^{2}$ \\ ${ }^{1}$ Slovak Medical University, Bratislava, Slovakia \\ ${ }^{2}$ Institute of Molecular Biology, Slovak Academy of Sciences, \\ Bratislava, Slovakia
}

\begin{abstract}
A mould Aspergillus versicolor clearly dominates in damp and mouldy indoor environments under Slovak dwelling/public building conditions (up to $1 / 3$ of all isolates during the last decade's surveys). Nearly all of its isolates are able to synthesize a mycotoxin sterigmatocystin (detected by LC/MS-MS), that showed severe in vitro as well as in vivo toxic potential in animal experiments (after intratracheal instillation to rats). In vitro toxicity of complex chloroformextractable endo- and exometabolites of 10 indoor, and related outdoor, $A$. versicolor isolates from a heavily mouldy kids' fashion store in Slovakia with complaints from the occupants of irritation of their airways has been evaluated by a bioassay with tracheal organ cultures of one-day old chicks ( 20 microg of toxicants per $\mathrm{mL}$ of cultivation medium). In the in vivo experiments, respiratory toxicity of the same metabolite mixtures was tested in Wistar rats during three days. The inflammatory and cytotoxic biomarkers were then analyzed in bronchoalveolar lavage fluid. Searching for the fungus possible source, molecular epidemiological study of the isolates was performed using RAMP PCR. Strains colonizing the indoor walls of the shop were the highest correlated to the outdoor airborne ones (Pearson correlation 97\%). While indoor airborne isolates correlated to the strains growing on retailed clothes at the levels of 90 or $86 \%$ according to Pearson. All micromycetes produced secondary metabolites that ceased ciliary beating in tracheal epithelium in the organ cultures already in $24 \mathrm{hrs}$ of the activity, i.e. in the sense of the method used, they belong to strong toxicants. Two of the isolates tested also produced extrolites without toxic effects detectable by the method. The metabolites also showed certain cytotoxic
\end{abstract}


and inflammation-inducing effects that were in concentration depending on the animal experiments. It has been proven that toxin production in fungi depends not only on the species but may vary between every single isolate as well. The most important outcome of the study is that microscopic filamentous fungi present in the dwelling indoor environment under Slovak (Central European) building/housing conditions might produce compounds even with the potential to damage the airways of occupants, while children remain the most vulnerable population.

Keywords: dwellings, dampness, fungal toxic metabolites, airways, intratracheal instillation.

\section{Introduction}

While there remain many unresolved scientific questions, we do know that exposure to high levels of mould causes some illnesses in susceptible people. Sick building syndrome (SBS) is a term used for symptoms, such as runny nose, itchy eyes, sore throat, headaches, commonly associated with staying in buildings with poor indoor air quality. The importance of indoor fungal growth in this phenomenon continues to be evident and indoor fungi are not only a scientific issue anymore but they are also becoming the social one [1]. Recently, research is more focused on non-allergic mechanisms that may be inducing adverse health effects of indoor fungi stemming from such fungal metabolites as beta-D-glucan, mycotoxins and fungal volatile organic compounds (VOCs) [2, 3]. Humidity indoors is a major factor relating to symptoms of SBS [4].

Tuomi et al. [5] analyzed 17 mycotoxins from 79 bulk building materials collected from water-damaged buildings. Their results showed sterigmatocystin was present in $24 \%$ of the samples, trichothecenes in $19 \%$ of the samples, and citrinin in 3 samples. Aspergillus versicolor was found on most sterigmatocystincontaining samples, and Stachybotrys chartarum were found on the samples in which satratoxins were present. Residents who were exposed to toxigenic fungi in water-damaged buildings might suffer from many nonspecific symptoms, although the health effects of indoor molds can be inconsistent $[6,7]$.

The mould $A$. versicolor develops yellow, orange-yellow to yellow-green compact colonies, sporulation might be often poor. The optimal growth temperature ranges between 25 and 27 minimal is found at $6-9^{\circ} \mathrm{C}$, optimal water activity $\left(\mathrm{a}_{\mathrm{w}}\right)$ of the cultivation medium $0.78-0.98$. A versicolor fungal cells have an antigen structure similar to that of Penicillium glabrum. After one-month's inhalation of the spores, laboratory rats showed granulomatous lesions in lung tissue, localized mainly near to the bronchi [8]. Most of the relevant papers dealing with $A$. versicolor discuss the production of carcinogenic mycotoxin sterigmatocystin. The frequency of toxigenic strains in the fungus population is rather high, about 74\%, under various laboratory conditions. Indoor $A$. versicolor isolates cultivated on plasterboard at $25^{\circ} \mathrm{C}$ in wet chambers for 3 months released into the material chloroform extractable endo- and exometabolites able to cease tracheal ciliary beating in chicks similar to the effect of strigmatocystin [9]. From the indoor environment quality point of view, this mould belongs to 
so-called first colonizers, i.e. its common air-borne microorganism found in nonsterile spaces. For example, it was found in $32 \%$ of examined houses (air, walls) and in $50 \%$ of schools in Western Europe, but also in house dust in Saudi Arabia (15,000 colony forming units/g, 7.66\% of mycoflora) [10]. A. versicolor clearly dominates in damp and mouldy indoor environments under Slovak dwelling/public building conditions (up to $1 / 3$ of all isolates during last decade's surveys) [11]. Nearly all of the isolates are able to synthesize sterigmatocystin (detected by LC/MS-MS) [12].

In vitro toxicity of complex chloroform-extractable endo- and exometabolites of 10 indoor, and related outdoor, A. versicolor isolates from a heavily mouldy kids' fashion store in Slovakia with complaints from occupants of irritation of their airways has been evaluated by a bioassay with tracheal organ cultures of 1-d-old chicks'. In the in vivo experiments, respiratory toxicity of the same metabolite mixtures was tested after intratracheal instillation in Wistar rats while the inflammatory and cytotoxic biomarkers were analyzed in bronchoalveolar lavage fluid. Searching for the possible source of the fungus, molecular epidemiological study of the isolates was performed.

\section{Material and methods}

\subsection{Moulds and their molecular characterization}

The indoor/outdoor air of the building tested was sampled by the aeroscope (AAIR 010, Agea, Ltd., Prague, Czech Republic) to obtain its particular mycoflora. Isolated $A$. versicolor strains (airborne -5 indoor, 1 outdoor as well as from mouldy indoor surfaces' swabs -3 and 1 from an air-co filter), 10 in total, tab. 1 , were cultivated in the liquid Sabouraud medium with $20 \%$ sucrose and $2 \%$ yeast extract at $30^{\circ} \mathrm{C}$ for $10 \mathrm{~d}$. Chloroform extracts of the biomass and the cultivation medium yielded crude fungal endo- or exometabolites for toxicological experiments [11].

Table 1: $\quad$ Aspergillus versicolor isolates used in the study.

\begin{tabular}{|l|l|}
\hline Isolate Nr. & Origin \\
\hline 393 & Indoor air (by the air-co system) \\
\hline 394 & Dtto \\
\hline 382 & Indoor air (next to W.C.) \\
\hline 383 & Dtto \\
\hline 426 & Indoor air (meeting room) \\
\hline 372 & Outdoor air (roof) \\
\hline 366 & Indoor wall \\
\hline 400 & Goods (coat) \\
\hline 403 & Goods (trousers) \\
\hline 413 & Air-co filter \\
\hline
\end{tabular}


To find the probable source of fungal contamination, the molecular epidemiological method of RAMP PCR was explored. It is the new PCR method and the special PCR program, using two primers (T14 and K7) for the analysis, enabling more precious identification of microorganisms than other PCRs (RAPD, AFLP, REP, etc.). Fungal DNA was isolated from the biomass, grown as given above, by the DNeasy Tissue Kit (QIAGEN, Hilden, Germany). PCR products were analyzed electrophoretically and UV visualized. The program GelCompare II software (Applied Maths, Kortrijk, Belgium) helped to establish Pearson's correlations of the fungal genome similarity [13].

\subsection{In vitro toxicity of fungal metabolites}

The bioassay based upon the ability of toxicants $(20 \mathrm{microg} / \mathrm{mL}$ cultivation Eagle's medium with Earl's salts ) - endo-, exometabolites of all $A$. versicolor isolates characterized, a mycotoxin sterigmatocystin (Sigma, Ltd., St. Louis, USA) commonly produced by $A$. versicolor as positive control, and a solvent dimethylsulphoxid (DMSO) $2 \%$ as negative one, to cease ciliary beating in the tracheas of one-d-old chicks at $37^{\circ} \mathrm{C}$ and $5 \% \mathrm{CO}_{2}$ after $24,48,72 \mathrm{hrs}$, resp., was employed [14].

\subsection{In vivo toxicity of $A$. versicolor metabolites after intratracheal instillation}

Groups of 6 male Wistar rats (Velaz, Prague, Czech Republic), at about $200 \mathrm{~g}$ were exposed per toxicant - endo- and exometabolites (Aend, Aex) of $A$. versicolor Nr. 366. Animal treatment followed the Guidelines of the European convention for the Protection of Vertebrate Animals for Experimental Purposes. Each rat was intratracheally instilled with 4 microg of the metabolite in $0.2 \mathrm{~mL}$ of $0.2 \%$ DMSO. Animals in the negative control group received only the solvent, or sterigmatocystin (StDAS) in the positive one. After 3-day's exposure, the animals were killed by exsanguination under thiopental anaesthesia $(150 \mathrm{mg} / \mathrm{kg}$ b. w.). Bronchoalveolar lavage was performed (5x), the pooled fluid (BALF) centrifuged and BALF cells isolated. Cytotoxic (phagocytic activity and viability of alveolar macrophages - AMs, the lactate dehydrogenase and acid phosphatase activities) and inflammatory response biomarkers (total BALF cell and AM counts, and white blood cells' differentials) were measured $[15,16]$.

\section{Results and discussion}

\subsection{Molecular epidemiology of $A$. versicolor strains}

The fungal isolates from indoor walls and outdoor air were highly correlated (Pearson's correlation 97\%). Indoor airborne isolates showed 90 or $86 \%$ correlation with those from mouldy textile or between different sampling positions (fig. 1). 

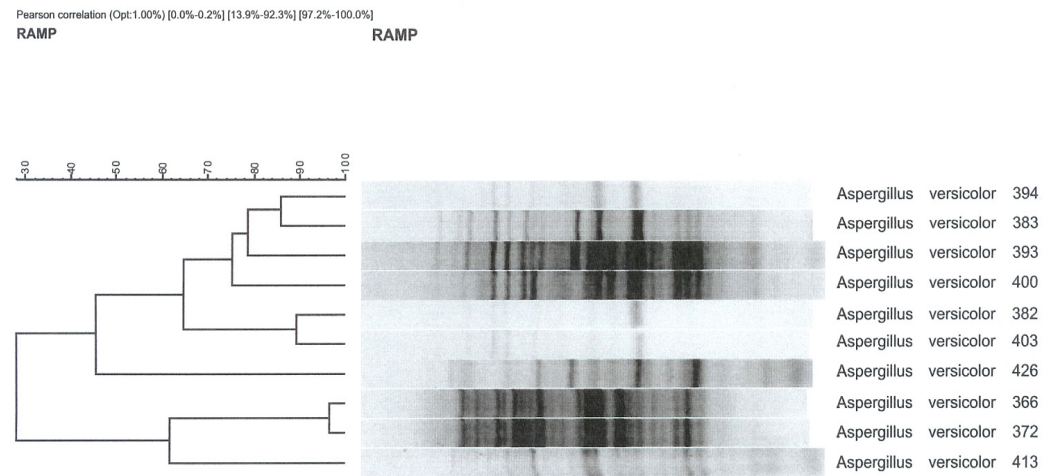

Figure 1: $\quad$ Pearson's correlation of Aspergillus versicolor isolates.

The highest correlation of $97 \%$ between $A$. versicolor strains could point to a mixing of out- and indoor air in the course of ventilation, while the outdoor air was apparently the source of the fungus later colonizing the walls inside the building examined.

On the other hand, mouldy textile items (trousers) could be seen as vectors carrying other, molecularly different, fungal strains into the indoor atmosphere as those correlated very well (90\% according to Pearson). It might be stated indoor and outdoor mycoflora clearly affects each other and it is impossible to distinguish between them precisely in terms of present fungal propagules [17].

\section{In vitro toxic potential of $A$. versicolor metabolites}

All fungal isolates tested produced mixtures of secondary chloroform-extractable metabolites able to stop movement of chicken tracheal cilia in 24 hrs, i.e., according to the method performed, they might be pronounced as strongly toxic. In previous studies (e.g. [12]), A. versicolor was recognized as the almost absolute producer of the mycotoxin sterigmatocystin and its derivatives. Sterigmatocystin was able to break down the ciliary beating in any such experiment formerly (e.g. [18]). So, this mycotoxin was also supposed to be the active toxic principle in chemically non-characterized mixtures of fungal products studied now.

Two indoor airborne strains (Nr. 383 and 394) also produced extrolites not damaging tracheal epithelium detected by the bioassay used. Therefore, it was again proven that toxin production does not belong to fungal species necessarily, but it may vary among single isolates. A. versicolor was found on most sterigmatocystin containing samples that represented $24 \%$ of building materials collected from water/damaged buildings and analyzed for mycotoxin content [5]. 


\subsection{Analysis of acute pulmonary toxicity of $A$. versicolor}

Pulmonary exposition to fungal exo- and endometabolites during $72 \mathrm{hrs}$ after intratracheal instillation to the rats did not cause statistically significant changes in some inflammatory parameters detected in bronchoalveolar lavage fluid (BALF). Though, total cell count and alveolar macrophages' (AMs) count in 1 $\mathrm{mL}$ BALF were elevated in the group of animals exposed to aspergillus metabolites (endo- and exometabolites - Aend, Aex in the graphs) comparing to the positive control group (exposed to the standard mycotoxin - StDAS in the graphs) (figs. 2 and 3). Differential counts of inflammation activated cells (AMs, lymphocytes and polymorphonuclears) were not altered by toxicants vs. controls in this experiment (figs. 4-6). Young monocytic AMs and binuclears were relatively depressed by fungal metabolites when compared to the positive control (fig. 7 and 8).

Results also indicated just very mild cytotoxic damage expressed as viability and phagocytic activity of AMs that were lowered only comparing to the negative control (DMSO in the graphs) (figs. 9 and 10). Activity of a cytoplasmatic enzyme lactate dehydrogenase (LDH) and the lysosomal ones acidic phosphatise (ACP) and cathepsin D (CATD) slightly increased in absolute numbers while the changes remained statistically insignificant, (figs. 11-13).

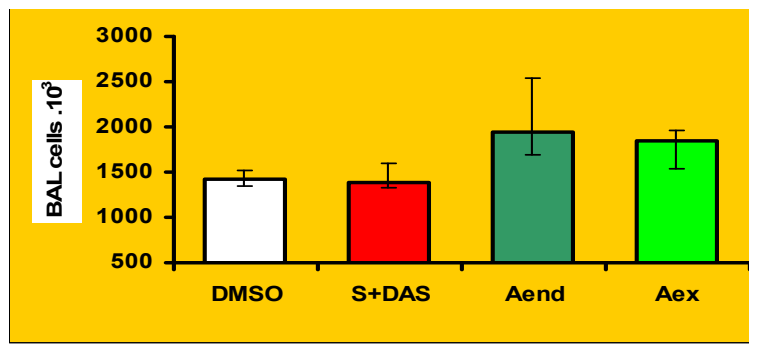

Figure 2: $\quad$ Total cells' count in bronchoalveolar lavage fluid (BALF).

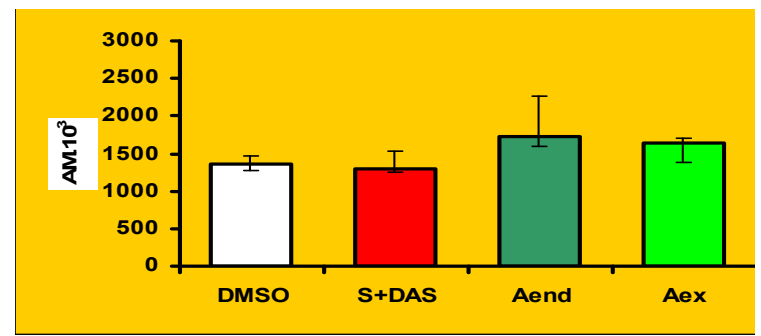

Figure 3: Alveolar macrophage (AM) count in BALF. 


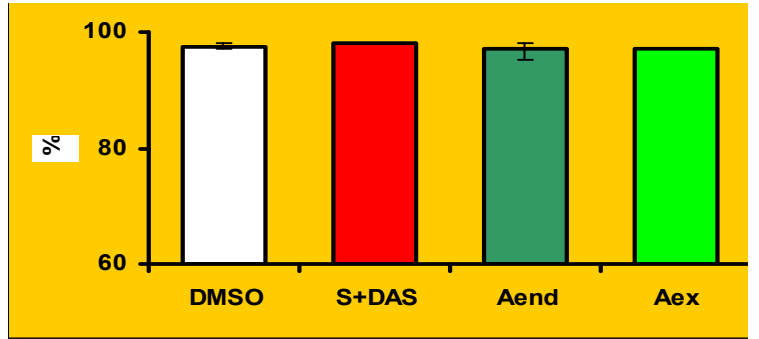

Figure 4: $\quad$ Proportion of AMs in total BALF cells.

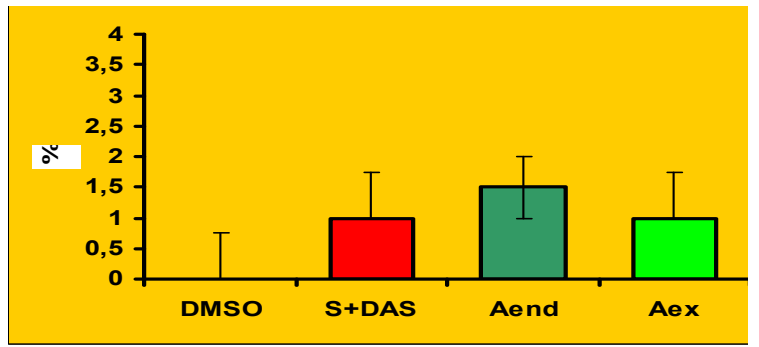

Figure 5: $\quad$ Proportion of lymphocytes in BALF cells.

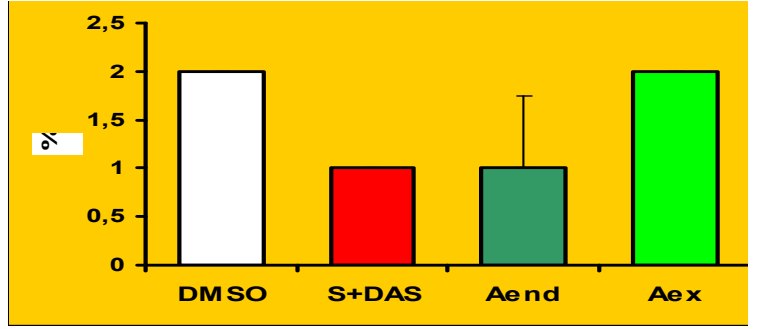

Figure 6: Proportion of polymorphonuclears in BALF cells.

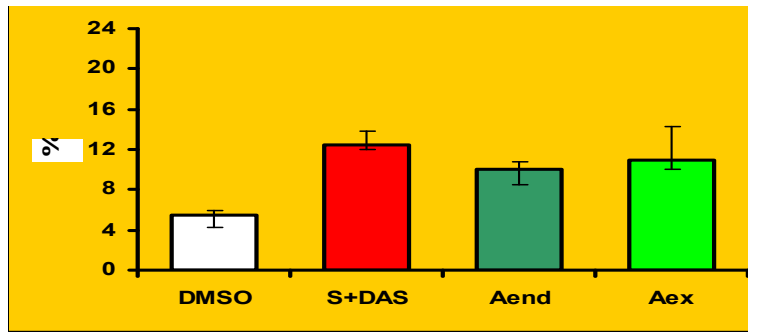

Figure 7: Proportion of young AMs in BALF cells. 


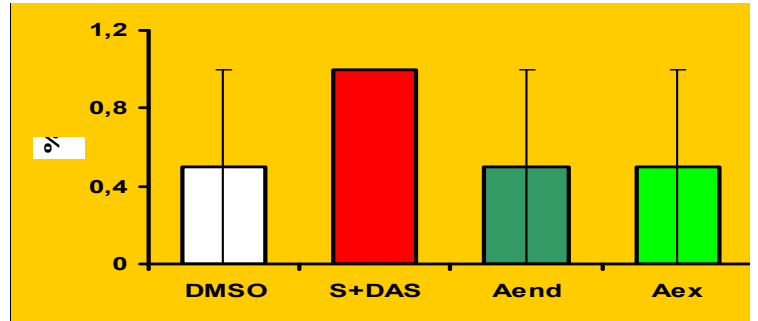

Figure 8: $\quad$ Proportion of binucleate cells in BALF cells.

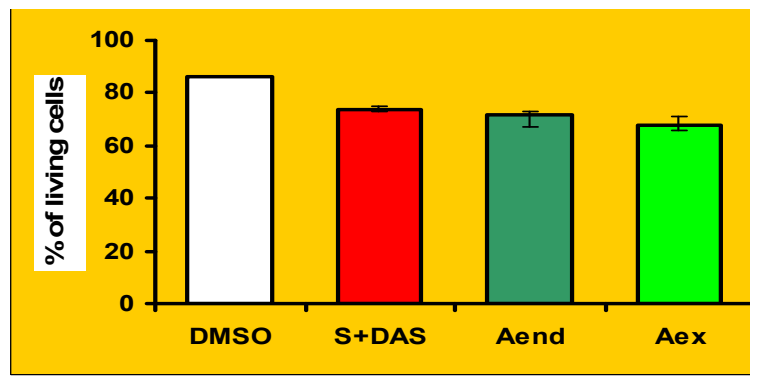

Figure 9: $\quad$ Viability of AMs.

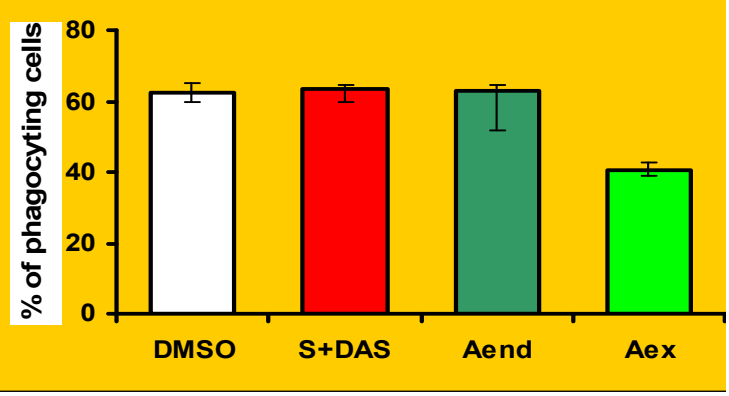

Figure 10: Phagocytic activity of AMs.

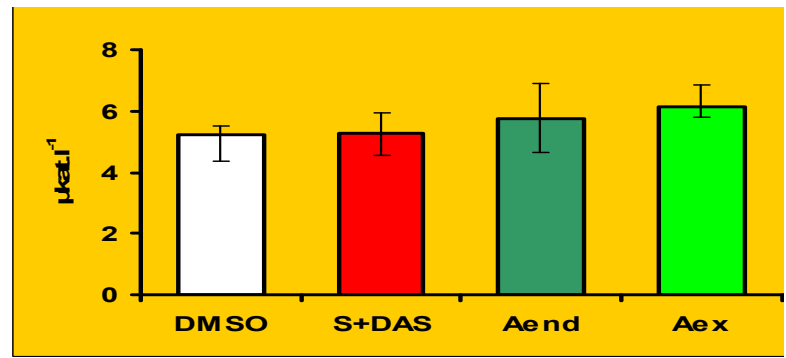

Figure 11: Activity of lactate dehydrogenase in cell free BALF. 


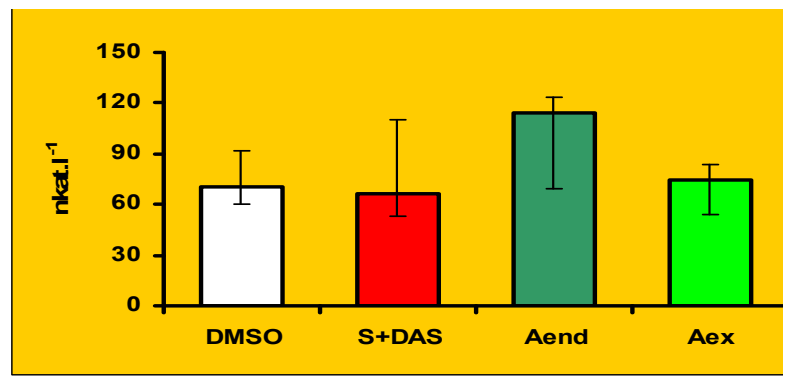

Figure 12: Activity of acidic phosphatase in cell free BALF.

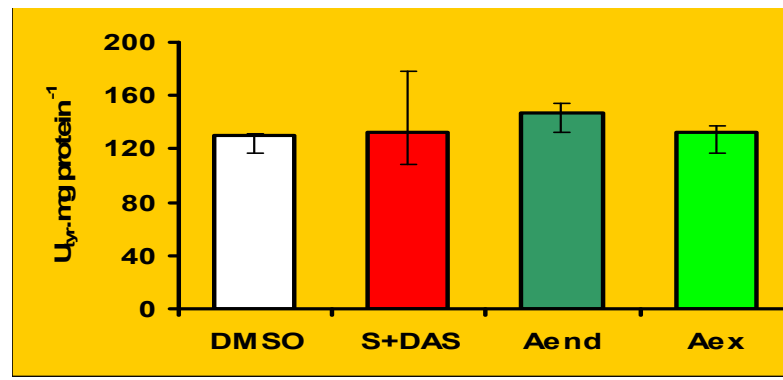

Figure 13: Activity of cathepsin D in cell free BALF.

It seems that $A$. versicolor metabolites tested possess less strong toxic proinflammatory and cytotoxic potential than similar complex mixtures produced by Stachybotrys chartarum, which were analyzed under identical experimental conditions prior to them: when the exposed rats showed significant remarks of cyttooxic damage - higher lactate dehydrogenase and acidic phosphatase activities in the cell free BALF, higher phagocytic activity and lower AMs viability as well as increased total BALF cell count, indicating inflammation, lower AM count and depressed granulocyte count related to the BALF cells. The significant increase in young AM proportion was probably related to significantly lower AM viability, which was either a secondary effect of inflammation or the consequence of metabolite cytotoxicity. Binucleate cell count may rise after long-term exposure to some environmental toxicants (tobacco smoke, some dusts etc.) and along with multinucleate cell count in lung suspension may well reflect chronic inflammation. In all of our studies so far the changes in these cell counts were not pronounced very well as the experiments took only $3 \mathrm{~d}$ and the inflammation was still acute $[15,16]$.

\section{Conclusion}

Physiological effects in vivo of sterigmatocystin or complex mixtures of $A$. versicolor metabolites following direct respiratory intake have not been described yet. We found they were able to initiate certain non-specific 
inflammatory response in the complex airways of experimental animals based on toxic reactions. Such response in the lungs may also cause systemic effects because of the spread of inflammatory cytokines in the blood to other organs of the entire body. Thus, it cannot be said yet that mycotoxin production in the buildings infested by fungi is definitely responsible for the ill health of their occupants, but it is clear that exposure to these chemicals should be avoided maximally.

\section{References}

[1] Straus, D.C., Consequences of mold exposure in buildings. Texas Journal of Rural Health, 19(1), pp. 8-13, 2001.

[2] Li, D.-W. \& Yang, C.S., Fungal contamination as a major contributor to sick building syndrome. Advances in Applied Microbiology. Vol. 55, ed. D.C. Straus, Elsevier: San Diego, pp. 31- 112, 2004.

[3] Shelton, B.G., Kirkladen, K.H., Flanders, W.D. \& Morris, G.K., Profiles of airborne fungi in buildings and outdoor environments in the United States. Applied and Environmental Microbiology, 68(6), pp. 1743-1753, 2002.

[4] Wieslander, G. \& Rylander, R., Humid buildings. Indoor Building Environment, 12(2), pp. 209 - 262, 2003.

[5] Tuomi, T., Reijula, K., Johnson, T., Hemminki, K., Hintikka, E.-L., Lindroos, O., Kalso, S., Koukila-Kahkola, P., Mussalo-Rahamaa, H. \& Haahtela, T., Mycotoxins in crude building materials from water damaged buildings. Applied and Environmental Microbiology, 66(6), pp. 1899-1904, 2000 .

[6] Ammann, H.M., ACGIH TLV Statement on bioaerosols; American Council of Government Industrial Hygienists. Bioaerosols, fungi and mycotoxins: health effects, assessment, prevention and control, ed. E. Johanning, ENYOEHC: Albany, pp. 520-521, 1999.

[7] Hessel, P.A., Klaver, J., Michaelchuk, D., McGhan, S., Carson, M.M. \& Melvin, D., The epidemiology of childhood asthma in Red Deer and Medicine Hat, Alberta. Canadian Respiratory Journal, 8(2), pp. 139-146, 2001.

[8] Piecková, E., Aspergillus sp. in dwellings and health implications of indoor fungi, www.aspergillus.man.ac.uk, 2001.

[9] Piecková, E. \& Jesenská, Z., Indoor fungi in dwellings. (In Slovak). Hygiena, 43(4), pp. 174-187, 1998.

[10] Piecková, E. \& Jesenská, Z., Microscopic fungi in dwellings and their health implications in humans. Annals of Agricultural and Environmental Medicine, 6(1), pp.1-11, 1999.

[11] Piecková, E. \& Kolláriková, Z.: In vitro toxicity of indoor moulds from Slovak dwellings. Environmental Toxicology II, eds. A.G. Kungolos, C.A. Brebbia \& M. Zamorano, WitPress: Southampton, pp. 227-234, 2008.

[12] Bloom, E., Bal, K., Nzman, E., Must, A. \& Larsson, L., Mass spectrometry/based strategy for direct detection and quantification of some mycotoxins produced by Stachybotrys and Aspergillus spp. in indoor 
environments. Applied and Environmental Microbiology, 73(5), pp. 42114217, 2007.

[13] Pangallo, D., Chovanová, K., Šimonovičová, A. \& Ferianc, P., Investigation of microbial community isolated from indoor artworks and air environment: Identification, biodegradative abilities, and DNA typing. Canadian Journal of Microbiology, 55(1) pp. 1-11, 2009.

[14] Jesenská, Z. \& Bernát, D., Effects of mycotoxins on in vitro movement of tracheal cilia from one-day-old chicks. Folia Microbiologica, 39(4), pp. 155-158, 1994.

[15] Piecková, E., Hurbánková, M., Černá, S., Pivovarová, Z. \& Kováčiková, Z., Pulmonary cytotoxicity of secondary metabolites of Stachybotrys chartarum (Ehrenb.) Hughes. Annals of Agricultural and Environmental Medicine, 13(2), pp. 259-262, 2006.

[16] Piecková, E., Hurbánková, M., Černá, S., Líšková, A., Kováčiková, Z., Kolláriková, Z. \& Wimmerová, S., Inflammatory and haematotoxic potential of indoor Stachybotrys chartarum (Ehrenb) Hughes metabolites. Archives of Industrial Hygiene and Toxicology, 60(4), pp. 401-410, 2009.

[17] Piecková, E. \& Pivovarová, Z., Indoor air fungal contamination in Slovak dwellings vs. outdoor aero-mycroflora. Proc. of the. 11th Int. Conf. Cold Climate, HVAC -ABOK: Moscow, pp. 230-234, 2006.

[18] Piecková, E. \& Kunová, Z., Indoor fungi and their ciliostatic metabolites. Annals of Agricultural and Environmental Medicine, 9(1), pp. 59-63, 2002. 